\title{
Characterization of Nivalenol-Producing Fusarium asiaticum That Causes Cereal Head Blight in Korea
}

\author{
Ja Yeong Jang ${ }^{1}$, Seul Gi Baek ${ }^{1}$, Jung-Hye Choi ${ }^{1}$, Sosoo Kim ${ }^{1}$, Jeomsoon Kim ${ }^{1}$, Da-Woon Kim ${ }^{2}$, \\ Sung-Hwan Yun (iD ${ }^{2 *}$, and Theresa Lee (iD) ${ }^{1 *}$ \\ ${ }^{1}$ Microbial Safety Team, National Institute of Agricultural Sciences, Wanju 55365, Korea \\ ${ }^{2}$ Department of Medical Biotechnology, Soonchunhyanag University, Asan 31538, Korea
}

(Received on June 18, 2019; Revised on August 14, 2019; Accepted on August 26, 2019)

Fusarium asiaticum of the $F$. graminearum species complex causes head blight in small-grain cereals. The nivalenol (NIV) chemotypes of $F$. asiaticum is more common than the deoxynivalenol (DON) chemotypes of $F$. asiaticum or $F$. graminearum in Korea. To understand the prevalence of $F$. asiaticum-NIV in Korean cereals, we characterized the biological traits of 80 cereal isolates of $\boldsymbol{F}$. asiaticum producing NIV or 3-acetyl-deoxynivalenol (3-ADON), and $54 \mathrm{~F}$. graminearum with 3-ADON or 15-acetyl-deoxynivalenol (15-ADON). There was no significant difference in mycelial growth between the chemotypes, but $F$. asiaticum isolates grew approximately $30 \%$ faster than $F$. graminearum isolates on potato dextrose agar. Sexual and asexual reproduction capacities differed markedly between the two species. Both chemotypes of $F$. graminearum (3-ADON and 15-ADON) produced significantly higher numbers of perithecia and conidia than $F$. asiaticum-NIV. The highest level of mycotoxins

\footnotetext{
*Corresponding author.

Theresa Lee

Phone) +82-63-238-3202, FAX) +82-63-238-3836

E-mail) tessyl1@korea.kr

Sung-Hwan Yun

Phone) +82-41-530-1288, FAX) +82-41-530-3085

E-mail)sy14@sch.ac.kr

ORCID

Theresa Lee

https://orcid.org/0000-0002-8230-650X

Sung-Hwan Yun

https://orcid.org/0000-0003-3438-6637

(c) This is an Open Access article distributed under the terms of the Creative Commons Attribution Non-Commercial License (http:// creativecommons.org/licenses/by-nc/4.0) which permits unrestricted noncommercial use, distribution, and reproduction in any medium, provided the original work is properly cited.
}

Articles can be freely viewed online at www.ppjonline.org. (sum of trichothecenes and zearalenone) was produced by $F$. graminearum-3-ADON on rice medium, followed by $F$. graminearum-15-ADON, F. asiaticum-3ADON, and $F$. asiaticum-NIV. Zearalenone levels were correlated with DON levels in some chemotypes, but not with NIV levels. Disease assessment on barley, maize, rice, and wheat revealed that both $F$. asiaticum and $F$. graminearum isolates were virulent toward all crops tested. However, there is a tendency that virulence levels of $F$. asiaticum-NIV isolates on rice were higher than those of $F$. graminearum isolates. Taken together, the phenotypic traits found among the Korean $F$. asiaticum-NIV isolates suggest an association with their host adaptation to certain environments in Korea.

Keywords : cereal, chemotype, head blight, mycotoxin, pathogenicity

Handling Editor : Shim, Won-Bo

Fusarium head blight (FHB) of small-grain cereals is a worldwide problem. The pathogens of FHB not only cause serious plant diseases, but also threaten public health via mycotoxin contamination of the infected crops (Goswami and Kistler, 2004). FHB mycotoxins include trichothecenes such as deoxynivalenol (DON), nivalenol (NIV), and their acetyl derivatives, and zearalenone (ZEA). These metabolites are toxic to humans and animals when consumed (Desjardins, 2006). The best-known causative agent of FHB, Fusarium graminearum sensu stricto (hereafter $F$. graminearum), is distributed worldwide, and belongs to the $F$. graminearum species complex (FGSC) consisting of at least 16 phylogenetically distinct species (Aoki et al., 2012). The distributions of some FGSC species and trichothecene chemotypes are regional. While $F$. gra- 
minearum is cosmopolitan (van der Lee et al., 2015; YliMattila 2010), F. asiaticum, which is a member of FGSC, is relatively confined to eastern Asia (Karugia et al., 2009; Lee et al., 2010; Qui et al., 2014; van der Lee et al., 2015; Zhang et al., 2010). Other FGSC members, F. aethiopicum and $F$. acacia-earnsii are reported to be endemic to the Southern hemisphere (Aoki et al., 2012; van der Lee et al., 2015).

While $F$. asiaticum is morphologically indistinguishable from $F$. graminearum (O'Donnell et al., 2004), the species dramatically differ in their toxin profiles. Although both species produce type B trichothecenes including DON, $\mathrm{NIV}$, and their acetyl derivatives, $F$. asiaticum is frequently associated with NIV chemotype, while $F$. graminearum is with DON chemotype (especially 15 -acetyl-DON [15ADON] chemotype) in Korea. F asiaticum-NIV has been also reported in Brazil, China, Japan, Nepal, and USA (Del Ponte et al., 2015; Desjardins and Proctor 2011; Gale et al., 2011; Gomes et al., 2015; Suga et al., 2008) on barley, maize, rice, and wheat. Barley and wheat are the crops frequently infected with $F$. asiaticum-NIV in China and Japan, while rice is affected in Brazil and USA (van der Lee et al., 2015), and maize in Nepal (Desjardins and Proctor 2011). In Korea, F. asiaticum-NIV has been isolated mostly from barley, rice and wheat at $95 \%$ frequencies (Lee et al., 2010; Shin et al., 2018; unpublished data). F. asiaticum-NIV is also present on maize, but less often than is $F$. graminearum-15-ADON. The first official outbreak of FHB was reported in 1963 (Chun 1963; Chung 1975). However, the fungal pathogen producing NIV was first reported in 1985 (Lee et al., 1985). The uneven distribution of FGSC species/chemotypes is found among crops and regions throughout the country. Neither the route nor timing of the introduction of $F$. asiaticum-NIV into Korea is known, but head blight outbreaks in small-grain cereals caused by this pathogen have long threatened both growers and consumers.

In an effort to further understand prevalence of $F$. asiaticum-NIV in Korean cereals, we characterized the biological properties of $F$. asiaticum-NIV and other FGSC species/chemotypes present in Korea. We compared $F$. asiaticum and $F$. graminearum isolates with DON or NIV chemotypes for their significant traits such as mycelial growth, sexual/asexual reproduction, pathogenicity, and toxin production.

\section{Materials and Methods}

Fungal resources. Fusarium isolates were retrieved from laboratory collections stored in $25 \%$ (v/v) glycerol at $-80^{\circ} \mathrm{C}$. All were single-spore isolates from barley, maize, rice, soybeans, or wheat grains collected from fields during 2014 and 2015 harvest, as previously described (Lee et al., 2016). For comparison with Korean strains, nine foreign $F$. asiaticum isolates were used. Seven of them were obtained from the Agriculture Research Service Culture Collection (ARS) and two were isolated directly from Japanese brown rice, as described previously (Lee et al., 2016). Freezedried ARS-isolates were revived on potato dextrose agar (PDA). All isolates were stored at $-80^{\circ} \mathrm{C}$. Species and Btype trichothecene genotypes were respectively determined based on PCR assays targeting the translation elongation factor 1-alpha $(T E F-1 \alpha)$ and TRI12 genes, as described previously by Starkey et al. (2007). Phylogenetic analyses were performed on three genes (TEF-1 $\alpha, M A T 1-1-3$, and TRI101), as described previously by Lee et al. (2016).

For isolation of Fusarium spp. from rice residues, rice straws were collected three times during each period between January and February, and between April and May in 2017, respectively, in rice paddy field located at Asan, Chungnam, Korea. A $10 \mathrm{~cm}$-long rice straw was placed on the Fusarium selective pentachloronitrobenzene medium (Nash and Synder, 1962) without surface sterilization, and incubated for 3-4 days. The species identification of the Fusarium isolates from rice straws were done using their nucleotide sequences of $T E F-1 \alpha$ obtained as described above.

Mycelial growth, conidiation, and perithecia production. Mycelial growth was evaluated on PDA and dichloran 18\% (v/v) glycerol agars (DG18, Oxoid, Basingstoke, UK). Five-day-old PDA cultures (5 $\mathrm{mm}$ in diameter) were center-placed on fresh PDA and DG18 plates and incubated at $25^{\circ} \mathrm{C}$. The PDA colony diameter was measured 3 and 7 days later, and the DG18 colony diameter was assessed at 7 and 14 days. The experiments were repeated three times and the average values were generated. Conidia were generated in carboxymethyl cellulose (CMC) broth cultures $(1.5 \% \mathrm{CMC}, 0.1 \%$ yeast extract, $0.05 \%$ $\mathrm{MgSO}_{4} \cdot 7 \mathrm{H}_{2} \mathrm{O}, 0.1 \% \mathrm{NH}_{4} \mathrm{NO}_{3}, 0.1 \% \mathrm{KH}_{2} \mathrm{PO}_{4}$ ) (Joo et al., 2015). After 5-day incubation at $25^{\circ} \mathrm{C}$ and $140 \mathrm{rpm}$, cultures were filtered through Miracloth (22-25 $\mu \mathrm{m}$ pore size; Merck Millipore, Billerica, MA, USA) to remove mycelia. Conidia in filtrates were counted using a hemocytometer (Superior Marienfeld, Bad Mergentheim, Germany). To measure perithecia production, PDA cultures $(5 \mathrm{~mm}$ in diameter) of each strain were placed in the centers of carrot agar prepared in $60 \mathrm{~mm}$ of the diameter Petri dishes (Leslie and Summerell, 2006). After 7 days of incubation at $25^{\circ} \mathrm{C}$, aerial mycelia were flooded with $1 \mathrm{ml}$ of $2.5 \%$ (v/v) Tween 
60 (Sigma-Aldrich, Dorset, UK) and then scraped with the back of a scalpel (Bowden and Leslie, 1999). The plates were then re-incubated under fluorescent light $(12 \mathrm{~h} /$ day $)$ for 7 days. The number of perithecia was quantified under a stereo microscope (Leica DMC2900, Wetzlar, Germany) at $2 \times$ resolution. The full image covered $8 \times 6 \mathrm{~mm}$ and three areas per plate were counted. Three plates per isolate were used to average the number of perithecia.

Mycotoxin analysis. Trichothecene and ZEA levels were prepared, harvested and measured in triplicate during fungal growth in rice medium as described previously (Lee et al., 2016) with minor modifications $(6 \mathrm{ml}$ water with $10 \mathrm{~g}$ long-grain rice). For NIV analysis, $2 \mathrm{~g}$ of ground rice cultures were extracted with $20 \mathrm{ml}$ water at $300 \mathrm{rpm}$ for $1 \mathrm{~h}$, centrifuged at 3,600 rpm for $10 \mathrm{~min}$, and filtered through Whatman No. 4 paper (Whatman, Kent, UK). The filtrate $(5 \mathrm{ml})$ was cleaned up using a DON-NIV WB immunoaffinity column (Vicam, Watertown, MA, USA). The eluates were subjected to liquid chromatography-mass spectrometry. DON, 3-acetyl-DON (3-ADON), 15-ADON, and ZEA were measured using a modified QuEChERS method (Lee et al., 2013). Milled samples (1 g) were extracted with $3.5 \mathrm{ml}$ water $/ 0.1 \%(\mathrm{v} / \mathrm{v})$ formic acid and $5 \mathrm{ml}$ acetonitrile $(\mathrm{ACN})$ at $300 \mathrm{rpm}$ for $1 \mathrm{~h}$. Then, $\mathrm{NaCl}(1 \mathrm{~g})$ and $\mathrm{MgSO}_{4}$ $(4 \mathrm{~g})$ were added, followed by vigorous shaking for $1 \mathrm{~min}$. The mixtures were centrifuged at 3,600 rpm for $10 \mathrm{~min}$, and $2 \mathrm{ml}$ aliquots of the supernatants were extracted with primary secondary amine $(50 \mathrm{mg})$ and $\mathrm{MgSO}_{4}(150 \mathrm{mg})$ with vortexing for $1 \mathrm{~min}$. The mixtures were centrifuged at 3,600 rpm for $10 \mathrm{~min}$, and $1 \mathrm{ml}$ amounts of supernatant were evaporated to dryness under a nitrogen stream. The residues were dissolved in $15 \%(\mathrm{v} / \mathrm{v}) \mathrm{MeOH}$, passed through a $0.2-\mu \mathrm{m}$ pore-sized filter, and analyzed via highperformance liquid chromatography (HPLC; e2695 separation module, Waters, Milford, MA, USA) fitted with a Zorbax SB-Aq C18 column (5 $\mu \mathrm{m}$ internal diameter, $3.0 \times$ $150 \mathrm{~mm}$; Agilent, Santa Clara, CA, USA). Mass spectrometry was performed using a Waters 3100 instrument operating in electrospray ionization mode. The detector was operated in the negative ionization mode, except when 15ADON was quantified. The quantification limits for NIV, DON, 3-ADON, 15-ADON, and ZEA were all $0.03 \mu \mathrm{g} /$ g. The recovery rates of NIV, DON, 3-ADON, 15ADON, and ZEA were 76.0, 109.0, 85.0, 62.2, and 116.0\%, respectively. Standard solutions of NIV, DON, 3-ADON, 15ADON, and ZEA in ACN were purchased from Biopure (Tulln, Austria). All organic solvents including $\mathrm{MeOH}$ and ACN were of HPLC grade (Fisher Scientific, Waltham, MA, USA). Tap water was transformed into MilliQ-quality water using a Millipore purification system.

Disease assessment. Pathogenicity of fungal isolates was evaluated as previously described (Lee et al., 2016) using susceptible cultivars of wheat (cv. Keumkang), barley (cv. Dahyang), rice (cv. Dongjin), and maize (cv. Kwangpyeongok) grown in a greenhouse. Prior to inoculation of the plants, fungal strains were cultured in CMC broth for 5 days for conidial production and the final conidial concentrations were adjusted to $1 \times 10^{4} / \mathrm{ml}$. For barley, rice and wheat, conidial suspensions were sprayed onto 5 head flowers from different pots. Each head was then covered with plastic bags for 3 days to prevent drying, and maintained in a greenhouse for 3 weeks. A negative control received only water. Pathogenicity was assessed as a percentage of symptomatic spikelets/head, as described previously (Groth et al., 1999). For maize, plant internodes (at the R0 stage, thus showing ear development) were stabbed with toothpicks dipped in conidial suspensions $\left(1 \times 10^{4} / \mathrm{ml}\right)$ as described previously (Lee et al., 2016), and the toothpicks remained in place throughout the experiment. Control stalks received only buffer. After 3 weeks, the inoculated internodes were cut and lesional lengths were measured. Disease was assessed as a rate of the lesional length/whole length of the inoculated internode. We used two plants/six internodes per isolate.

Statistics. Statistical significance was assessed using the Duncan multiple range test and Student's $t$-test. Analyses were performed using the SPSS statistical package (version 21.0, IBM Corp., Armonk, NY, USA). We also calculated $95 \%$ confidence intervals. Correlation coefficients were calculated with Microsoft Excel 2010.

\section{Results}

We tested a total of 134 isolates consisting of $80 \mathrm{~F}$. asiaticum and $54 \mathrm{~F}$. graminearum among which three chemotypes (NIV, 3-ADON, and 15-ADON) were found (Table $1)$. All NIV types were $F$. asiaticum $(n=66)$ and all 15 ADON types $(n=33)$ were $F$. graminearum; 3 -ADON types were found in both species. None of isolates belonging to $F$. asiaticum-15-ADON or $F$. graminearum-NIV type was identified. Phylogenetic analyses revealed that all isolates examined were clearly separated into two phylogenetically distinct clades, each was matched to the species, but thetrichothecene chemotypes were not associated with speciation; they were dispersed evenly within each species (Supplementary Fig. 1). 
Table 1. Number of isolates tested in this study

\begin{tabular}{|c|c|c|c|c|c|}
\hline \multirow{3}{*}{ Host } & \multicolumn{4}{|c|}{ Chemotype } & \multirow{3}{*}{ Total } \\
\hline & \multirow{2}{*}{$\begin{array}{c}\text { NIV } \\
\mathrm{Fa}\end{array}$} & \multicolumn{2}{|c|}{ 3-ADON } & \multirow{2}{*}{$\frac{15-\mathrm{ADON}}{\mathrm{Fg}}$} & \\
\hline & & $\mathrm{Fa}$ & $\mathrm{Fg}$ & & \\
\hline Barley & $18(1)$ & $4(1)$ & 4 & 5 & $31(2)$ \\
\hline Maize & $12(1)$ & $1(1)$ & 5 & 11 & $29(2)$ \\
\hline Rice & $26(2)$ & 3 & 9 & 14 & $52(2)$ \\
\hline Soybean & 2 & 0 & 0 & 2 & 4 \\
\hline Wheat & 8 & $6(1)$ & 3 & 1 & $18(1)$ \\
\hline Unknown & (2) & & & & (2) \\
\hline Total & $66(6)$ & $14(3)$ & 21 & 33 & $134(9)$ \\
\hline
\end{tabular}

Number in parenthesis indicates foreign isolate.

NIV, nivalenol; 3-ADON, 3-acetyl-deoxynivalenol; 15-ADON, 15-acetyl-deoxynivalenol; Fa, Fusarium asiatium; Fg, Fusarium graminearum.

Mycelial growth, conidiation, and sexual reproduction. On both PDA and DG18 agar, the average growth rates of $F$. asiaticum isolates were higher than those of $F$. graminearum strains (Table 2). Of the various species/ chemotypes, $F$. asiaticum-3-ADON grew faster on both media than did other types, followed by $F$. asiaticum-NIV. However, no significant differences were found between species or chemotypes during later cultivation periods.

$F$. asiaticum exhibited poor sexual and asexual reproduction. In CMC liquid medium, $F$. graminearum (both 3-ADON and 15-ADON types) produced significantly higher numbers of conidia (approximately 4.5 -fold on average) than $F$. asiaticum $(P=0.05)$ (Table 3$)$. However, foreign $F$. asiaticum strains (of both NIV and 3-ADON types) produced more conidia ( $\sim 9.1$-fold on average) than Korean $F$. graminearum isolates. Of the $F$. asiaticum isolates, NIV types produced more conidia ( $\sim 25.8$-fold) than the 3-ADON types.

On carrot agar medium, $F$. graminearum-DON isolates produced approximately 20 times more perithecia than

Table 2. Comparison of mycelial growth (colony diameter) among Fusarium asiaticum and F. graminearum chemotypes

\begin{tabular}{|c|c|c|c|c|c|}
\hline \multirow{2}{*}{ Species } & \multirow{2}{*}{ Chemotypes $(n)$} & \multicolumn{2}{|c|}{ PDA (mm) } & \multicolumn{2}{|c|}{ DG18 (mm) } \\
\hline & & Day 3 & Day 7 & Day 7 & Day 14 \\
\hline \multirow[t]{3}{*}{ F. asiaticum } & Total (80) & $48.2 \pm 10.7 \mathrm{a}$ & $82.5 \pm 7.6 \mathrm{a}$ & $33.2 \pm 6.7 \mathrm{a}$ & $56.6 \pm 11.2 \mathrm{a}$ \\
\hline & NIV(66) & $47.4 \pm 10.5 \mathrm{a}$ & $82.2 \pm 8.3 \mathrm{a}$ & $32.8 \pm 7.1 \mathrm{ab}$ & $55.9 \pm 11.5 \mathrm{ab}$ \\
\hline & 3-ADON (14) & $52.1 \pm 11.1 \mathrm{a}$ & $84.3 \pm 1.5 \mathrm{a}$ & $35.2 \pm 4.3 \mathrm{a}$ & $59.7 \pm 9.5 \mathrm{a}$ \\
\hline \multirow{3}{*}{$\begin{array}{l}\text { F. asiaticum } \\
\text { (foreign origin) }\end{array}$} & Total (9) & $34.7 \pm 11.0 \mathrm{~b}$ & $69.0 \pm 21.6 b$ & $18.6 \pm 6.2 \mathrm{c}$ & $30.8 \pm 8.5 \mathrm{c}$ \\
\hline & NIV (6) & $35.0 \pm 26.3 b$ & $66.7 \pm 26.3 b$ & $19.6 \pm 7.2 \mathrm{c}$ & $33.3 \pm 9.1 \mathrm{c}$ \\
\hline & 3-ADON (3) & $34.2 \pm 4.0 \mathrm{~b}$ & $73.6 \pm 9.5 \mathrm{ab}$ & $16.6 \pm 3.9 \mathrm{c}$ & $25.9 \pm 4.9 \mathrm{c}$ \\
\hline \multirow[t]{4}{*}{$F$.graminearum } & Total (54) & $35.5 \pm 11.2 \mathrm{~b}$ & $76.5 \pm 12.8 \mathrm{a}$ & $28.3 \pm 5.1 \mathrm{~b}$ & $49.4 \pm 10.2 b$ \\
\hline & 3-ADON (21) & $37.1 \pm 11.0 \mathrm{~b}$ & $75.9 \pm 14.4 \mathrm{ab}$ & $29.7 \pm 5.5 \mathrm{ab}$ & $53.7 \pm 9.7 \mathrm{ab}$ \\
\hline & 15-ADON (33) & $34.5 \pm 11.4 \mathrm{~b}$ & $76.9 \pm 11.8 \mathrm{ab}$ & $27.3 \pm 4.8 \mathrm{~b}$ & $46.7 \pm 9.6 \mathrm{~b}$ \\
\hline & 15-ADON (PH-1) & $47.0 \pm 2.0$ & $74.0 \pm 6.0$ & $33.7 \pm 6.0$ & $46.7 \pm 6.0$ \\
\hline
\end{tabular}

Means with the same letter did not differ significantly $(P=0.05)$ and the sums were treated separately.

PDA, potato dextrose agar; DG18, dichloran 18\% glycerol agar; NIV, nivalenol; 3-ADON, 3-acetyl-deoxynivalenol; 15-ADON, 15-acetyldeoxynivalenol.

Table 3. Production of conidia and perithecia by Fusarium asiaticum and F. graminearum

\begin{tabular}{llcc}
\hline Species & Chemotype $(n)$ & Conidia $(104 / \mathrm{ml})$ & Perithecia $(8 \times 6 \mathrm{~mm})$ \\
\hline F. asiaticum & Total $(80)$ & $62.1 \pm 2.6 \mathrm{~b}$ & $8.5 \pm 28.9 \mathrm{~b}$ \\
& NIV $(66)$ & $74.7 \pm 214.2 \mathrm{c}$ & $10.1 \pm 31.5 \mathrm{~b}$ \\
& 3-ADON $(14)$ & $2.9 \pm 2.6 \mathrm{c}$ & $0.0 \pm 0.0 \mathrm{~b}$ \\
\hline F. asiaticum & NIV $(6)$ & $3,484.8 \pm 6,053.4 \mathrm{a}$ & $0.0 \pm 0.0 \mathrm{~b}$ \\
(foreign origin) & 3-ADON (3) & $1,653.6 \pm 2,782.6 \mathrm{~b}$ & $5.8 \pm 10.1 \mathrm{~b}$ \\
\hline F. graminearum & Total $(54)$ & $282.3 \pm 472.5 \mathrm{a}$ & $212.0 \pm 213.0 \mathrm{a}$ \\
& 3-ADON $(21)$ & $402.1 \pm 659.5 \mathrm{c}$ & $177.8 \pm 213.1 \mathrm{a}$ \\
\hline F. graminearum PH-1 & 15-ADON (33) & $206.1 \pm 287.3 \mathrm{c}$ & $233.7 \pm 211.3 \mathrm{a}$ \\
\hline
\end{tabular}

Means with the same letter did not differ significantly $(P=0.05)$ and the sums were treated separately.

NIV, nivalenol; 3-ADON, 3-acetyl-deoxynivalenol; 15-ADON, 15-acetyl-deoxynivalenol. 


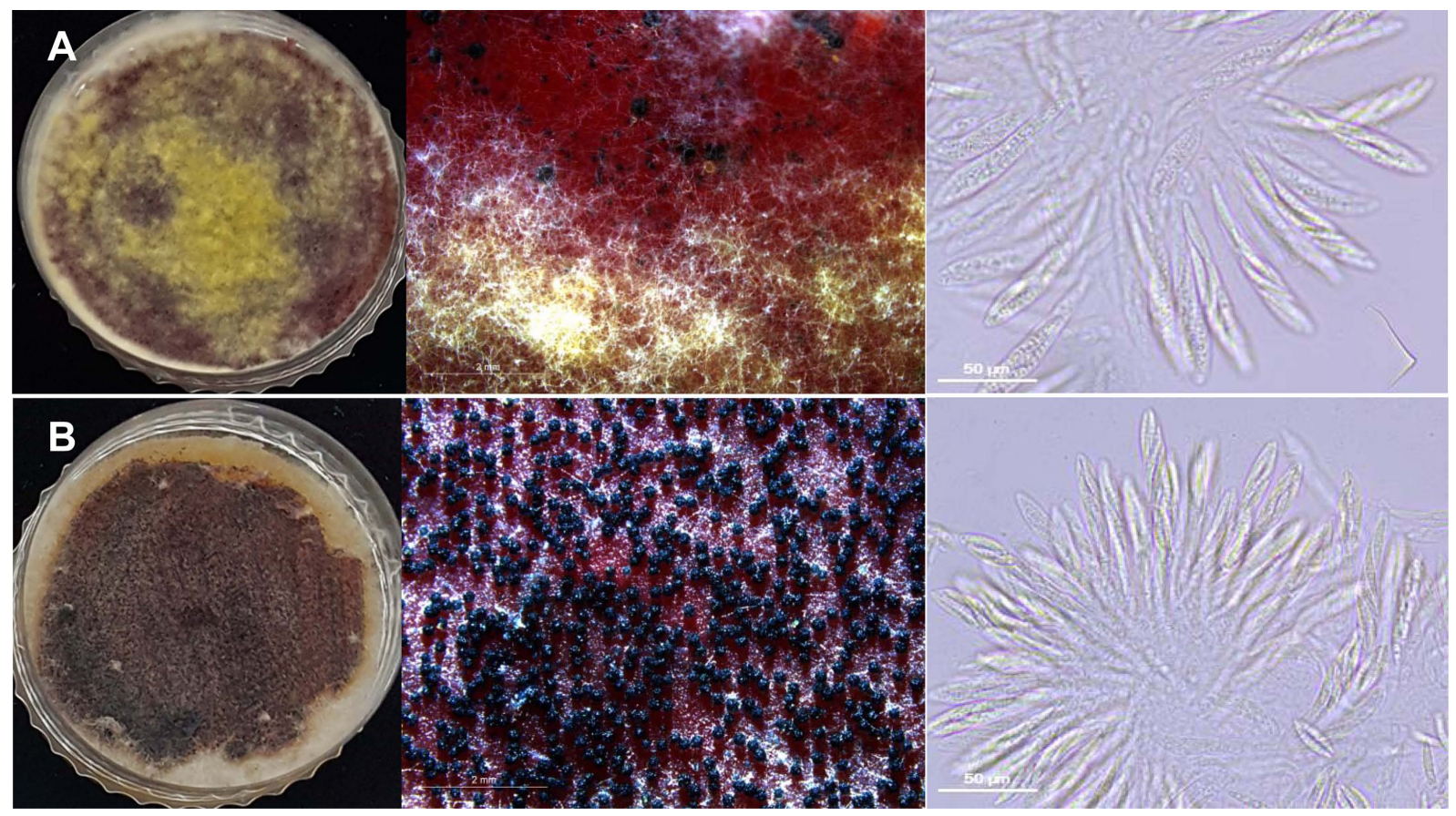

Fig. 1. Comparison of perithecia produced by Fusarium asiaticum (A) and Fusarium graminearum (B) on artificial medium. From left to right, perithecia produced on carrot agar at 2 weeks after inoculation; enlarged $(2 \times)$ surface of the 2-week culture plate; and asci released from perithecia $(400 \times)$. Scale bars $=50 \mu \mathrm{m}$. (A) F. asiaticum 15RhGg04-f2. (B) F. graminearum 15ShGw06-f1.

either DON or NIV type isolates of $F$. asiaticum (Table 3, Fig. 1). Of the $F$. asiaticum isolates, Korean NIV and foreign 3-ADON types produced much fewer perithecia, while the other types produced none. Despite the poor capacity for sexual reproduction in $F$. asiaticum, all perithecia were full of normal asci containing good-looking ascospores (Fig. 1). Of the $F$. graminearum isolates, both types produced similar number of perithecia.

Mycotoxin production. F. asiaticum-NIV produced the least amounts of trichothecene toxins among the species/ chemotypes tested. Excluding the NIV isolates, the sum levels of DONs (including 3-ADON) produced by $F$. asiaticum-3-ADON were all less than those produced by
$F$. graminearum chemotypes (Table 4 ). The NIV levels produced by $66 F$. asiaticum-NIV strains ranged from 0.05 to $16.1 \mu \mathrm{g} / \mathrm{g}$ (mean $6.5 \mu \mathrm{g} / \mathrm{g}$ ) (Supplementary Table 1). On average, rice isolates produced the most NIV $(7.2 \mu \mathrm{g} / \mathrm{g})$, followed by the wheat, soybean, barley, and maize $(5.8 \mu \mathrm{g} /$ g) strains. Of the six foreign $F$. asiaticum-NIV strains, only three produced NIV $(8.2-12.2 \mu \mathrm{g} / \mathrm{g})$.

Most DON-type isolates produced both 3-ADON and 15-ADON along with DON, regardless of species. $F$. asiaticum-3-ADON isolates produced mean levels of 50.1 $\mu \mathrm{g} / \mathrm{g}$ DON, $14.1 \mu \mathrm{g} / \mathrm{g} 3-\mathrm{ADON}$, and $6.5 \mu \mathrm{g} / \mathrm{g} 15-\mathrm{ADON}$; foreign $F$. asiaticum-3-ADON isolates produced up to $13.6 \mu \mathrm{g} / \mathrm{g}$ DON in total (Table 4, Supplementary Table 2). In contrast, $F$. graminearum-3-ADON isolates produced

Table 4. Mean level of mycotoxins produced by all chemotypes of Fusarium asiaticum (Fa) and F. graminearum (Fg) tested in this study

\begin{tabular}{|c|c|c|c|c|c|c|c|c|c|}
\hline \multirow{2}{*}{ Species } & \multirow{2}{*}{ Chemotype } & \multirow{2}{*}{ No. } & \multicolumn{7}{|c|}{ Toxin $(\mu \mathrm{g} / \mathrm{g})$} \\
\hline & & & NIV & DON & 3-ADON & 15-ADON & DON total & ZEA & Total \\
\hline \multirow[t]{2}{*}{$\mathrm{Fa}$} & NIV & 66 & $6.6 \pm 4.2$ & - & - & - & - & $22.1 \pm 43.0 \mathrm{~b}$ & $28.7 \pm 43.6 b$ \\
\hline & 3-ADON & 14 & - & $50.1 \pm 41.7 \mathrm{~b}$ & $14.1 \pm 7.0 \mathrm{~b}$ & $6.5 \pm 3.5 b$ & $70.6 \pm 47.0 \mathrm{~b}$ & $64.9 \pm 80.3 b$ & $135.5 \pm 117.2 b$ \\
\hline \multirow[t]{2}{*}{$\mathrm{Fg}$} & 3-ADON & 21 & - & $261.3 \pm 216.0 \mathrm{a}$ & $111.3 \pm 100.4 \mathrm{a}$ & $69.4 \pm 109.3 \mathrm{a}$ & $441.9 \pm 386.7 \mathrm{a}$ & $417.9 \pm 468.9 \mathrm{a}$ & $859.8 \pm 581.3 \mathrm{a}$ \\
\hline & 15-ADON & 33 & - & $221.2 \pm 176.0 \mathrm{a}$ & $6.1 \pm 23.6 \mathrm{~b}$ & $57.5 \pm 46.0 \mathrm{a}$ & $284.8 \pm 190.7 \mathrm{a}$ & $396.1 \pm 502.4 \mathrm{a}$ & $680.9 \pm 614.7 \mathrm{a}$ \\
\hline
\end{tabular}

Means with the same letter did not differ significantly $(P=0.05)$.

NIV, nivalenol; 3-ADON, 3-acetyl-deoxynivalenol; 15-ADON, 15-acetyl-deoxynivalenol; ZEA, zearalenone. 
5-fold more DON, 5-fold more 3-ADON, and 10-fold more $15-\mathrm{ADON}$ than $F$. asiaticum-3-ADON isolates. For the 3-ADON types of both species, the 3-ADON levels were nearly twice the 15-ADON levels, while the 3-ADON level $(6.1 \mu \mathrm{g} / \mathrm{g})$ of $F$. graminearum-15-ADON was 9-fold less than that of 15-ADON. Although the total level of DON by $F$. graminearum-3-ADON types were 1.5 times higher than that of $F$. graminearum-15-ADON types, the difference was not supported statistically.

ZEA production levels also varied among species/chemotype. ZEA production by DON types was greater than that by NIV types, and $F$. graminearum produced more ZEA than $F$. asiaticum (Table 4 ). The ZEA level produced by $F$. asiaticum-NIV was again the lowest among all the tested species/chemotypes. Among the $F$. asiaticum-NIV types, maize isolates produced the highest level of ZEA $(57.0 \mu \mathrm{g} /$ $\mathrm{g}$ for mean, up to $239 \mu \mathrm{g} / \mathrm{g})$, followed by barley $(16.8 \mu \mathrm{g} / \mathrm{g})$, rice, wheat, and soybean $(4.2 \mu \mathrm{g} / \mathrm{g})$ isolates (Supplementary Table 1). Foreign $F$. asiaticum-NIV strains produced the amounts similar to domestic ones (up to $254.3 \mu \mathrm{g} / \mathrm{g}$ ). $F$. asiaticum-3-ADON produced about three-fold more ZEA on average than the $F$. asiaticum-NIV. Barley isolates of $F$. asiaticum-3-ADON produced the most ZEA (mean 87.4 $\mu \mathrm{g} / \mathrm{g})$, followed by the rice $(69.2 \mu \mathrm{g} / \mathrm{g})$ and wheat $(58.5$ $\mu \mathrm{g} / \mathrm{g}$ ) isolates (Supplementary Table 2). Single maize isolate of $F$. asiaticum-3-ADON did not produce any toxins, whereas a single foreign maize strain of the same type produced $532.8 \mu \mathrm{g} / \mathrm{g}$ ZEA, which level is exceptionally higher than other toxins. The highest level of ZEA was produced by the $F$. graminearum-3-ADON type $(417.9 \mu \mathrm{g} / \mathrm{g})$, followed by the $F$. graminearum-15-ADON $(390.2 \mu \mathrm{g} / \mathrm{g})$. Of the $F$. graminearum strains, the highest ZEA producer was a soybean $15-A D O N$ type isolate $(2,331.4 \mu \mathrm{g} / \mathrm{g})$, followed by a rice $3-\mathrm{ADON}$ isolate $(2,217.3 \mu \mathrm{g} / \mathrm{g})$.

In some chemotypes, ZEA level showed correlation with DON or ADON levels but not with NIV levels. In 3-ADON types of both species from barley, ZEA levels were correlated with either DON (correlation coefficient of 0.9056 for $F$. asiaticum and 0.6759 for $F$. graminearum) or with total DON ( 0.8639 for $F$. asiaticum and 0.767 for $F$. graminearum). Correlations were also found between the ZEA and 3-ADON for $F$. graminearum-15-ADON types from rice (0.9706), $F$. asiaticum-3-ADON from wheat (0.6748), and $F$. graminearum-3-ADON from barley (0.659). Correlations between ZEA and 15-ADON were shown in $F$. graminearum-3-ADON from barley $(0.7675)$ and $F$. asiaticum-3-ADON from wheat (0.7305).

Pathogenicity on cereals. Most isolates were virulent toward all the cereals such as barley, maize, rice, and wheat. Most $F$. asiaticum-NIV isolates were virulent toward all cereals and the virulence levels appeared similar to or even higher than the other species/chemotypes (Table 5). Regardless of host origins, all $F$. asiaticum-NIV isolates examined were more virulent toward barley than the other cereals. The average virulence levels of all $18 \mathrm{~F}$. asiaticumNIV isolates on barley were ranging from $53 \%$ to $87 \%$, while those on maize (14 isolates tested), rice (6 isolates), and wheat (19 isolates) were $19-31 \%, 6.1-22.1 \%$, and 0.7 $35 \%$, respectively (Supplementary Table 3 ). Similarly, $F$. asiaticum-3-ADON isolates were also more virulent toward barley than the other cereals. The average virulence levels on barley (5 isolates tested) were $41-47 \%$, while those on maize (4 isolates), rice ( 2 isolates), and wheat (2 isolates) were $15-20 \%, 14 \%$, and $2.2-5.4 \%$, respectively (Supplementary Table 3).

Unlike $F$. asiaticum isolates, the average virulence levels of both 3-ADON and 15-ADON $F$. graminearum chemotype isolates toward barley, maize, and wheat seemed to be more varied among isolates than those of $F$. asiaticum isolates (Supplementary Table 3). Interestingly, the virulence levels $(2.6-2.0 \%)$ of three out of four $F$. graminearum isolates tested toward rice seemed to be much lower than those of $F$. asiaticum isolates. All foreign $F$. asiaticum

Table 5. Mean level of pathogenicity on cereals by all chemotypes of Fusarium asiaticum (Fa) and F. graminearum (Fg) tested in this study

\begin{tabular}{|c|c|c|c|c|c|}
\hline \multirow{2}{*}{ Species } & \multirow{2}{*}{ Chemotype } & \multicolumn{4}{|c|}{ Pathogenicity $^{a}$} \\
\hline & & Barley & Maize & Rice & Wheat \\
\hline \multirow[t]{2}{*}{$\mathrm{Fa}$} & NIV & $60.4 \pm 12.7(18)$ & $23.0 \pm 10.0(14)$ & $23.7 \pm 32.0(7)$ & $20.1 \pm 23.5(19)$ \\
\hline & 3-ADON & $44.0 \pm 23.2(5)$ & $16.8 \pm 3.6(4)$ & $14.1 \pm 16.3(2)$ & $3.8 \pm 2.3(2)$ \\
\hline \multirow[t]{2}{*}{$\mathrm{Fg}$} & 3-ADON & $50.5 \pm 28.8(5)$ & $26.7 \pm 13.7(5)$ & $2.6(1)$ & $16.6 \pm 16.2(4)$ \\
\hline & 15-ADON & $53.4 \pm 70.0(2)$ & $23.2 \pm 8.9(4)$ & $5.6 \pm 4.8(3)$ & $22.2 \pm 33.1(4)$ \\
\hline
\end{tabular}

NIV, nivalenol; 3-ADON, 3-acetyl-deoxynivalenol; 15-ADON, 15-acetyl-deoxynivalenol.

${ }^{a}$ Pathogenicity toward barley, rice and wheat was measured as average percentage (\%) of diseased spikelets per head. Maize was measured as an average length of lesion ( $\mathrm{mm}$ ) on the stalk. The number of isolate tested in parenthesis. 
Table 6. Number of Fusarium isolates obtained from rice residues (straws) in 2017

\begin{tabular}{lrccccccc}
\hline & \multicolumn{7}{c}{ Pusarium species $^{\text {a }}$} & \multicolumn{7}{c}{ Total } \\
\cline { 2 - 7 } & $F a$ & $F g$ & $F e i$ & $F c$ & $F a r$ & $F o$ & \\
\hline Jan-Feb & 2 & 1 & 14 & 1 & 4 & 1 & 24 \\
Apr-May & 15 & - & 11 & - & - & - & 26 \\
\hline
\end{tabular}

$F a, F$. asiaticum; $F g, F$. graminearum; Fei, F. equiseti-incarnatum species complex; Fc, F. commune; Far, F. armeniacum; Fo, F. oxysporum; -, not isolated.

${ }^{a}$ Rice straw samples were collected three times during each period.

isolates were virulent toward barley and, to a lesser extent, maize. Two foreign $F$. asiaticum-3-ADON isolates were aggressive to rice as much as the $F$. asiaticum-NIV barley isolate.

Isolation of Fusarium spp. from rice straws during winter and spring. Several species of Fusarium were recovered from the rice straws collected from the same rice paddy field during winter and spring (Table 6). The total numbers of Fusarium isolates obtained from two different seasons were similar to each other, but frequencies of each Fusarium species in the samples were dramatically different. In winter samples, the fungal isolates belonging to the $F$. equiseti-incarnatum species complex were predominant with $58.3 \%$ (14 out of all 24 Fusarium isolates), while FGSC isolates ( $F$. asiaticum and $F$. graminearum) were recovered with a lower frequency $(12.5 \%, 3$ out of 24$)$; three other species $(F$. commune, $F$. armeniacum, and $F$. oxysporum) were also found. However, $F$. asiaticum was predominant species in spring samples with $57.7 \%$ (15 out of 26), followed by $F$. equiseti-incarnarum with $42.3 \%$. Interestingly, the three other species recovered from the winter samples were not found in the spring samples (Table $6)$.

\section{Discussion}

This is the first report to explore Korean $F$. asiaticum and $F$. graminearum populations with different trichothecene chemotypes in terms of their mycological, toxicological, and pathological characteristics. We found that $F$. asiaticumNIV type isolates differed from other species/chemotypes isolates tested, particularly exhibiting low capacity of asexual/sexual reproduction and mycotoxin production. Similar work was carried out by Liu et al. (2017) using the three Chinese chemotypes ( $F$. asiaticum-NIV and-3-ADON, $F$. graminearum-15-ADON), where $F$. graminearum15-ADON produced the highest quantities of perithecia. Our work was also confirmed by an earlier study (Nicolli et al., 2018) using the chemotypes of Brazil (15-ADON of $F$. graminearum, 3-ADON and NIV of other FGSC members rather than $F$. graminearum or $F$. asiaticum). However, when perithecia production was compared, two Chinese $F$. asiaticum chemotypes (NIV and 3-ADON) appeared to produce more perithecia than the respective Korean chemotypes. Previous work on Korean isolates also showed that $F$. asiaticum was 10 -fold less self-fertile than $F$. graminearum (Lee et al., 2009). All these results together indicate that capacity for sexual reproduction by Korean $F$. asiaticum may be intrinsically low, characterizing a regional population. However, it is intriguing that $F$. asiaticum survived better than $F$. graminearum during winter by vigorously producing perithecia on rice residues in more restrictive environment (Lee et al., 2009). Hence, it is possible that $F$. asiaticum may engage in sexual reproduction under certain environmental conditions. However, no natural occurrence of perithecia in rice fields have been reported in Korea where both rice and barley have been rotating. Therefore, it requires further investigations to re-confirm the superior capability of $F$. asiaticum over $F$. graminearum for perithecia production (Lee et al., 2009).

In addition, relatively poor capacities of both NIV and 3-DON chemtotypes of Korean $F$. asiaticum isolates for asexual/sexual reproduction strongly suggest that these traits, which are recognized as important characteristics of survival and dispersal in $F$. graminearum in the fields, are no longer essential in Korea. This hypothesis is possible as other propagules (e.g., mycelia) of $F$. asiaticum-NIV can survive on previous crop residues (e.g., rice straws) during Korean winter. As rice fields are rotated consecutively with barley or wheat mostly in southern part of Korea, such farming practices could allow pathogens to mingle and adapt in the same fields over many years. The recovery of $F$. asiaticum isolates from rice straws collected during winter would support this possibility. Based on a higher mycelial growth rate on PDA and prevalence on spring rice straws, it can be presumed that capacity of Korean $F$. asiaticum isolates for mycelial growth would be a fitness trait especially on rice.

The NIV levels produced in vitro by $F$. asiaticum isolates were lower than the DON levels produced by either species under the laboratory. Previous work done by Gale et al. (2011) showed the similar result that the NIV types of both $F$. asiaticum and $F$. graminearum produced 4-fold less trichothecenes than the DON types. This indicates that the NIV biosynthesis machinery per se may be weaker than that of DON in the genus Fusarium. This may be due to the difference in biosynthetic pathways between NIV and DON; the synthesis of NIV requires two more enzymatic 
steps catalyzed by TRI13 and TRI7, respectively, compared to that of DON (Lee et al., 2002). Further work with more NIV types of other FGSC species, including $F$. cortaderiae, $F$. luisianense, $F$. meridionale, and $F$. vorosii, will be necessary to test this hypothesis. In addition, $F$. asiaticum-NIV types produced less amounts of ZEA than other isolates, further suggesting that the mycotoxin biosynthetic machinery (at least for NIV and ZEA) of $F$. asiaticum is functionally different from that of $F$. graminearum. The strong correlations $(>0.9)$ between ZEA and DONs levels in some isolates were contrary to the previous study reporting a negative correlation between DON and ZEA levels (Tancic et al., 2015), suggesting further studies. Although $F$. asiaticum-NIV frequently produce both NIV and ZEA simultaneously, there was no correlation found between ZEA and NIV levels.

It is notable that the Korean 3-ADON chemotypes (of both species) produced approximately twice amounts of 3-ADON on average than those of 15-ADON, while the $15-\mathrm{ADON}$ chemotypes ( $F$. graminearum) produced much less 3-ADON. Thus Korean 3-ADON chemotypes resulted in production of more DONs than 15-ADON under the laboratory condition. A study with Chinese populations, however, showed that $F$. asiaticum-3-ADON produced more 15-ADON than 3-ADON (Liu et al., 2017). The different results from two countries showed that even the same chemotypes have different capacities developed under the respective environments. As 3-ADON is more toxic and aggressive on host plants than 15-ADON producer (Ward et al., 2008; Zhang et al., 2010, 2012), monitoring for a change or increase in the frequency of 3-ADON chemotype should be conducted.

Korean $F$. asiaticum, predominantly found in rice or barley, is more associated with NIV type than DON type. Thus, it is reasonable to focus on how NIV production is associated with their pathogenicity and fitness on these crops in Korea. Although rice may afford a selective growth advantage to $F$. asiaticum at some phase of the fungal life cycle (Lee et al., 2009), it is not known if NIV would confer a pathogenic advantage in Korean $F$. asiaticum isolates. Unlike NIV, DON is known to be one of the virulence factors toward wheat (Proctor et al., 2002), but not toward barley or maize (Maier et al., 2006). However, it is not clear if DON also serves as a virulence factor toward rice, as is the case for wheat. Based on our virulence test results, we hypothesize that NIV, but not DON, is a virulence factor in Korean $F$. asiaticum-NIV isolates particularly on rice. Note that all $F$. asiaticum-NIV isolates examined were aggressive to four crops including rice with similar degree, while $F$. graminaerum-DON isolates showed a lower virulence only on rice when compared to $F$. asiaticum-NIV isolates. Given that NIV production contributed to fungal virulence toward maize (Maier et al., 2006), it is possible to speculate that NIV is toxic to all four crops as much as DON, but DON is less only to rice than NIV. In this perspective, it would be not so surprised that the Korean $F$. asiaticum-NIV isolates keep a low NIV production level assuming that NIV requires more energy for biosynthesis than DON. To test the role of NIV in pathogenicity, it is necessary to perform further analyses including a virulence assay on rice with a transgenic $F$. asiatiumNIV strain lacking NIV production.

$F$. asiaticum-15-ADON and $F$. graminearum-NIV type appear to be rare in Asia including Korea, Japan, and China, although $F$. asiaticum-15-ADON was detected in $18 \%$ of wheat isolates from China (Puri et al., 2012). Of 237 FGSC isolates from Louisiana (USA), however, $F$. graminearum-NIV constituted $79 \%$ of all isolates (Gale et al., 2011). Fungal proportions appear to vary regionally so that continuous monitoring is required to track population changes. We speculate that uncommon species/chemotypes are either poorly pathogenic or are in the early stages of adaptation to their host regions.

In summary, the Korean $F$. asiaticum-NIV type isolates differed from other species/chemotypes isolated from the country, being aggressive toward several crops but highly associated with rice, producing low levels of NIV and ZEA, and engaging in less asexual/sexual reproduction than $F$. graminearum. These characteristics may explain why the $F$. asiaticum-NIV type prevails in Korea although further comprehensive investigations should be performed. They rely on vigorous mycelial growth and the synthesis of NIV as a virulence factor, but not on sexual reproduction for survival in rice paddy field. Hence, they may adapt with crops (e.g., rice) by maintaining the specific fitness traits that differ considerably from those of $F$. graminearum. However, it is not clear why the ability of $F$. asiaticumNIV types to produce conidia was lower than that of other species/chemotypes, assuming that conidia are the only primary inocula of $F$. asiaticum-NIV.

\section{Acknowledgments}

This study was carried out with the support of "Research Program for Agricultural Science \& Technology Development (Project No. PJ012485)", National Institute of Agricultural Sciences, Rural Development Administration, Republic of Korea, and was supported by the Strategic Initiative for Microbiomes in Agriculture and Food, Ministry of Agriculture, Food and Rural Affairs, Republic of Korea 
(918019-4).

\section{Electronic Supplementary Material}

Supplementary materials are available at The Plant Pathology Journal website (http://www.ppjonline.org/).

\section{References}

Aoki, T., Ward, T. J., Kistler, H. C. and O’Donnell, K. 2012. Systematics, phylogeny and trichothecene mycotoxin potential of Fusarium head blight cereal pathogens. Mycotoxins 62:91102.

Bowden, R. L. and Leslie, J. F. 1999. Sexual recombination in Gibberella zeae. Phytopathology 89:182-188.

Chun, J. H. 1963. Epidemiological survey of human mycotoxicosis caused by scabby cereals. In: Research report on wheat and barley scab, ed. by Republic of Korea, pp. 385-507. Ministry of Agriculture and Forestry, Seoul, Korea (in Korean).

Chung, H. S. 1975. Cereal scab causing mycotoxicoses in Korea and present status of mycotoxin researches. Korean. J. Mycol. 3:31-36.

Del Ponte, E. M., Spolti, P., Ward, T. J., Gomes, L. B., Nicolli, C. P., Kuhnem, P. R., Silva, C. N. and Tessmann, D. J. 2015. Regional and field-specific factors affect the composition of fusarium head blight pathogens in subtropical no-till wheat agroecosystem of Brazil. Phytopathology 105:246-254.

Desjardins, A. E. 2006. Fusarium mycotoxins: chemistry, genetics and biology. American Phytopathological Society, St. Paul, MN, USA. 268 pp.

Desjardins, A. E. and Proctor, R. H. 2011. Genetic diversity and trichothecene chemotypes of the Fusarium graminearum clade isolated from maize in Nepal and identification of a putative new lineage. Fungal Biol. 115:38-48.

Gale, L. R., Harrison, S. A., Ward, T. J., O’Donnell, K., Milus, E. A., Gale, S. W. and Kistler, H. C. 2011. Nivalenol-type populations of Fusarium graminearum and F. asiaticum are prevalent on wheat in southern Louisiana. Phytopathology 101:124-134.

Gomes, L. B., Ward, T. J., Badiale-Furlong, E. and Del Ponte, E. M. 2015. Species composition, toxigenic potential and pathogenicity of Fusarium graminearum species complex isolates from southern Brazilian rice. Plant Pathol. 64:980-987.

Goswami, R. S. and Kistler, H. C. 2004. Heading for disaster: Fusarium graminearum on cereal crops. Mol. Plant Pathol. 5:515-525.

Groth, J. V., Ozmon, E. A. and Busch, R. H. 1999. Repeatability and relationship of incidence and severity measures of scab of wheat caused by Fusarium graminearum in inoculated nurseries. Plant Dis. 83:1033-1038.

Joo, H. J., Kim, H.-Y., Kim, L.-H., Lee, S., Ryu, J.-G. and Lee, T. 2015. A Brevibacillus sp. antagonistic to mycotoxigenic Fusarium spp. Biol. Control 87:64-70.
Karugia, G. W., Suga, H., Gale, L. R., Nakajima, T., Tomimura, K. and Hyakumachi, M. 2009. Population structure of the Fusarium graminearum species complex from a single Japanese wheat field sampled in two consecutive years. Plant Dis. 93:170-174.

Lee, J., Chang, I. Y., Kim, H., Yun, S. H., Leslie, J. F. and Lee, Y. W. 2009. Genetic diversity and fitness of Fusarium graminearum populations from rice in Korea. Appl. Environ. Microbiol. 75:3289-3295.

Lee, S.-H., Lee, J.-K., Nam, Y.-J., Lee, S.-H., Ryu, J.-G. and Lee, T. 2010. Population structure of Fusarium graminearum from maize and rice in 2009 in Korea. Plant Pathol. J. 26:321-327.

Lee, S., Lee, T., Kim, M., Yu, O., Im, H. and Ryu, J.-G. 2013. Survey on contamination of Fusarium mycotoxins in 2011-harvested rice and its by-products from rice processing complexes in Korea. Res. Plant Dis. 19:259-264 (in Korean).

Lee, T., Han, Y.-K., Kim, K.-H., Yun, S.-H. and Lee, Y.-W. 2002. Tri13 and Tri7 determine deoxynivalenol- and nivalenolproducing chemotypes of Gibberella zeae. Appl. Environ. Microbiol. 68:2148-2154.

Lee, T., Paek, J.-S., Lee, K. A., Lee, S., Choi, J.-H., Ham, H., Hong, S. K. and Ryu, J.-G. 2016. Occurrence of toxigenic Fusarium vorosii among small grain cereals in Korea. Plant Pathol. J. 32:407-413.

Lee, U. S., Jang, H. S., Tanaka, T., Hasegawa, A., Oh, Y. J. and Ueno, Y. 1985. The coexistence of the Fusarium mycotoxins nivalenol, deoxynivalenol and zearalenone in Korean cereals harvested in 1983. Food Addit. Contam. 2:185-192.

Leslie, J. F. and Summerell, B. A. 2006. The Fusarium laboratory manual. Blackwell Publishing, Ames, IA, USA. 388 pp.

Liu, Y.-Y., Sun, H.-Y., Li, W., Xia, Y.-L., Deng, Y.-Y., Zhang, A.X. and Chen, H.-G. 2017. Fitness of three chemotypes of Fusarium graminearum species complex in major winter wheatproducing areas of China. PLoS ONE 12:e0174040.

Maier, F. J., Miedaner, T., Hadeler, B., Felk, A., Salomon, S., Lemmens, M., Kassner, H. and Schäfer, W. 2006. Involvement of trichothecenes in fusarioses of wheat, barley and maize evaluated by gene disruption of the trichodiene synthase (Tri5) gene in three field isolates of different chemotype and virulence. Mol. Plant Pathol. 7:449-461.

Nash, S. M. and Snyder, W. C. 1962. Quantitative estimations by plate counts of propagules of the Bean root rot Fusarium in field soils. Phytopathology 52:567-572.

Nicolli, C. P., Machado, F. J., Spolti, P. and Del Ponte, E. M. 2018. Fitness traits of deoxynivalenol and nivalenol-producing Fusarium graminearum species complex strains from wheat. Plant Dis. 102:1341-1347.

O’Donnell, K., Ward, T. J., Geiser, D. M., Kistler, H. C. and Aoki, T. 2004. Genealogical concordance between the mating type locus and seven other nuclear genes supports formal recognition of nine phylogenetically distinct species within the $F u$ sarium graminearum clade. Fungal Genet. Biol. 41:600-623.

Proctor, R. H., Desjardins, A. E., McCormick, S. P., Plattner, R. D., Alexander, N. J. and Brown, D. W. 2002. Genetic analysis 
of the role of trichothecene and fumonisin mycotoxins in the virulence of Fusarium. Eur. J. Plant Pathol. 108:691-698.

Puri, K. D., Saucedo, E. S. and Zhong, S. 2012. Molecular characterization of Fusarium head blight pathogens sampled from a naturally infected disease nursery used for wheat breeding programs in China. Plant Dis. 96:1280-1285.

Qui, J., Xu, J. and Shi, J. 2014. Molecular characterization of the Fusarium graminearum species complex in Eastern China. Eur. J. Plant Pathol. 139:811-823.

Shin, S., Son, J.-H., Park, J.-C., Kim, K.-H., Yoon, Y., Cheong, Y.-K., Kim, K.-H., Hyun, J.-N., Park, C. S., Dill-Macky, R. and Kang, C.-S. 2018. Comparative pathogenicity of Fusarium graminearum isolates from wheat kernels in Korea. Plant Pathol. J. 34:347-355.

Starkey, D. E., Ward, T. J., Aoki, T., Gale, L. R., Kistler, H. C., Geiser, D. M., Suga, H., Tóth, B., Varga, J. and O’Donnell, K. 2007. Global molecular surveillance reveals novel Fusarium head blight species and trichothecene toxin diversity. Fungal Genet. Biol. 44:1191-1204.

Suga, H., Karugia, G. W., Ward, T., Gale, L. R., Tomimura, K., Nakajima, T., Miyasaka, A., Koizumi, S., Kageyama, K. and Hyakumachi, M. 2008. Molecular characterization of the Fusarium graminearum species complex in Japan. Phytopathology 98:159-166.

Tančić, S., Stanković, S., Lević, J. and Krnjaja, V. 2015. Cor- relation of deoxynivalenol and zearalenone production by Fusarium species originating from wheat and maize grain. Pestic. Phytomed. 30:99-105.

van der Lee, T., Zhang, H., Diepeningen, A. and Waalwijk, C. 2015. Biogeography of Fusarium graminearum species complex and chemotypes: a review. Food Addit. Contam. Part A Chem. Anal. Control Expo. Risk Assess. 32:453-460.

Ward, T. J., Clear, R. M., Rooney, A. P., O'Donnell, K., Gaba, D., Patrick, S., Starkey, D. E., Gilbert, J., Geiser, D. M. and Nowicki, T. W. 2008. An adaptive evolutionary shift in Fusarium head blight pathogen populations is driving the rapid spread of more toxigenic Fusarium graminearum in North America. Fungal Genet. Biol. 45:473-484.

Yli-Mattila, T. 2010. Ecology and evolution of toxigenic Fusarium species in cereals in northern Europe and Asia. J. Plant Pathol. 92:7-18.

Zhang, H., van der Lee, T., Waalwijk, C., Chen, W., Xu, J., Xu, J., Zhang, Y. and Feng, J. 2012. Population analysis of the Fusarium graminearum species complex from wheat in China show a shift to more aggressive isolates. PLOS ONE 7:e31722.

Zhang, H., Zhang, Z., van der Lee, T., Chen, W. Q., Xu, J., Xu, J. S., Yang, L., Yu, D., Waalwijk, C. and Feng, J. 2010. Population genetic analyses of Fusarium asiaticum populations from barley suggest a recent shift favoring $3 \mathrm{ADON}$ producers in southern China. Phytopathology 100:328-336. 